hypercatecholaminemia. Plasma BNP is a sensitive and reliable cardiac biomarker for detection of cardiac involvement in children with severe EV71 infection.

\section{PO-0262 ANALYSIS OF PAEDIATRIC FORENSIC CASES; A SECOND LEVEL HOSPITAL EMERGENCY SERVICE EXPERIENCE}

${ }^{1} \mathrm{E}$ Çevik, ${ }^{2} \mathrm{~S}$ Kemer, ${ }^{3} \mathrm{~F}$ Karademir, ${ }^{4} \mathrm{YA}$ Acar. ${ }^{1}$ Emergency Department, Van Military Hospital, Van, Turkey; ${ }^{2}$ Department of Pediatrics, Van Military Hospital, Van, Turkey; ${ }^{3}$ Department of Pediatrics, GATA Haydarpasa Training Hospital, Istanbul, Turkey; ${ }^{4}$ Emergency Department, Etimesgut Military Hospital, Ankara, Turkey

10.1136/archdischild-2014-307384.916

Background and aims Childhood accidents are the leading cause of health problems, death and disability that can be prevented. Accidents can be predicted and avoided by identifying the risks. The aim of this study is to investigate the characteristics of paediatric forensic cases and to reveal the relationship between judicial decisions and trauma scores.

Methods Forensic reports of the Children under 18 admitted to our emergency department of Van Military Hospital, a second level hospital, between January and November 2013 were retrospectively evaluated. Demographic data of the patients and life threat decisions were investigated and trauma scores were calculated. P-value under 0,05 was considered to be significant.

Results Forty-four (15.1\%) of 290 forensic cases admitted to our emergency department were under 18. Of the patients, $43.2 \%$ (19) were female and the mean age was $8.7 \pm 5.41$ (minmax: $0-17)$. Ten $(22.7 \%)$ of them were traffic accident, 8 $(18.2 \%)$ were falling from height, $12(27.3 \%)$ were assault, 2 (4.5\%) were burn, $10(22.7 \%)$ were poisoning and suicide. Four of all patients $(9.1 \%)$ were having life threat and $9(20.5 \%)$ patients were having no injury requiring simple medical intervention. In the evaluation of the cases; Injury Severity Score (ISS) was $1.93 \pm 2.27$ (1-16), Revised Trauma Score (RTS) was $99.72 \pm 0.17$ (99-99.83), Trauma score- injury severity score (TRISS) was $7,082 \pm 0.15(7,108-7,841)$ and New Injury Severity Score (NITS) was $2.55 \pm 3.52$ (1-16).

Conclusions Traffic accidents, falls, assaults and poisonings are the most common forensic cases in childhood and we found a significant relationship between life threat decision and anatomical and physiological trauma scores.

\section{PO-0263 SAFETY NETTING IN PAEDIATRIC EMERGENCY CARE: A SYSTEMATIC REVIEW AND PROTOCOL DEVELOPMENT}

E Kerkhof, D Geurts, M Wiggers, HA Moll, R Oostenbrink. General Paediatrics, Erasmus University Medical Center - Sophia Children's Hospital, Rotterdam, Netherlands

\subsection{6/archdischild-2014-307384.917}

Background Safety netting strategies after emergency department (ED) discharge are mostly not standardised.

Aim To develop a safety netting protocol for children at risk for serious infections (SI) discharged from the ED.

Methods Patients: Children with fever, vomiting/diarrhoea or dyspnoea (1 month ${ }^{-1} 6$ years).

Design: Systematic Literature Review to Select Best Evidence for Determinants of ED-Revisits and Prospective Cohort Study

Setting: ED of Erasmus MC-Sophia Children's Hospital (May 2010-December 2012)
Intervention Standardised telephonic questionnaires on disease course three days after ED-discharge.

OutcomeED-Revisits with Intervention (Diagnostics or Treatment)

Analysis All determinants and time until ED-revisits were tested by multivariable logistic regression and Kaplan-Meier survival analysis.

Results (Preliminary) Follow-up data were available for 1366/ 1656(83\%) children, median age 21 months (IQR10-45), 57\% boys. $856(63 \%)$ were febrile children, $300(22 \%)$ children suffered from vomiting/diarrhoea and $210(15 \%)$ children had dyspnoea. Overall there were 401(29\%) revisits in which 54\% (n = 216) interventions were performed.

We confirmed the determinants: age $<1$ year, respiratory/circulatory symptoms and signs of dehydration, as identified in our systematic review, associated with ED-revisits. Additional, parental concern was a determinant for revisit in febrile children. Unscheduled revisits of children with vomiting/diarrhoea occurred mostly within one day and in febrile or dyspnoeic children within 2 days after initial ED-visit.

Discussion and conclusion We identified strong determinants of revisits by prospective data collection in ED-discharged children at risk for SI. We are preparing a multicentre efficacy study to use these determinants and time frames in ED safety netting management.

\section{PO-0264 CONSTIPATION TREATMENT PROTOCOL IN CRITICALLY ILL CHILDREN}

J López, SN Fernández, R González, MJ Solana, J Urbano, B Toledo, MJ Santiago, J del Castillo, S Mencía, J López-Herce. Pediatric Intensive Care Department, Gregorio Marañón University Hospital, Madrid, Spain

\subsection{6/archdischild-2014-307384.918}

Backgrounds and aims Constipation in critically ill patients is associated with severity of illness. There are no clinical guidelines in critically ill children. The aim of this study is to review the results with our treatment protocol.

Methods Prospective observational study including children admitted to the PICU $>3$ days. Constipation was defined as $\geq 4$ days with no stools. Constipation was treated with saline rectal enemas or polyethylene glycol + ions (PEGI). PEGI was prepared mixing one paediatric packet $(6.9 \mathrm{~g})$ with $20 \mathrm{ml}$ of water. Initial dose was $1-2 \mathrm{ml} / \mathrm{kg} / 8 \mathrm{~h}$ and after intestinal transit was observed, it was diminished to $0,5-1 \mathrm{ml} / \mathrm{kg} / 24 \mathrm{~h}$. Adult preparation form $(13,8 \mathrm{~g})$ in $40 \mathrm{ml}$ of water was used in children $>25$ $\mathrm{kg}$. Clinical and demographic data were recorded.

Results 68/150 patients (45.3\%), median age 38.5 months (IQR 8.5-82.5) had constipation. Rectal enemas were administered to 15 patients (19.2\%). It was useful (stool produced within the next two hours) in 50\% of them. 47 patients $(60.2 \%)$ were treated with PEGI obtaining stool production in the next $48 \mathrm{~h}$ in $60.3 \%$ of them. Median (IQR) dose of PGEI was $0.9(0.6-1.3 \mathrm{~g} / \mathrm{kg} /$ day). Median (IQR) time to first stool production after PEGI was 1 (0-2 days). Diarrhoea was the most frequent side effect observed in 4 patients with PEGI.

Conclusions Constipation in critically ill children is a very common problem. Our treatment protocol seems to be useful and secure. More studies are necessary to evaluate treatment efficacy and security and to develop clinical guidelines. 